\title{
Underground polymeric l-shaped pipeline vibrations under seismic effect
}

\author{
Nematilla Nishonov $^{1 *}$, Diyorbek Bekmirzaev ${ }^{1}$, Akbar Ergashov ${ }^{2}$, Ziyoviddin Rakhimjonov ${ }^{2}$ \\ and Asror Khurramov ${ }^{1}$ \\ ${ }^{1}$ Academy of Science of the Republic of Uzbekistan Institute of Mechanics and Seismic Stability of \\ Structures named after M.T. Urazbaev, Tashkent, Uzbekistan \\ ${ }^{2}$ Tashkent State Transport University, Tashkent, Uzbekistan
}

\begin{abstract}
The simultaneous equations of longitudinal and transverse vibrations of an underground polymeric L-shaped pipeline under the arbitrary direction of seismic load were derived in the paper. A computational scheme of the problem was constructed using central finitedifference relations. The analysis of the results obtained on the simultaneous longitudinal and transverse vibrations of underground polymeric L-shaped pipelines under seismic loading was conducted. The stress-strain state of the L-shaped polymeric pipeline subjected to seismic effect was determined, and the axial forces and bending moments arising in curved pipelines during an earthquake were determined.
\end{abstract}

\section{Introduction}

The issues of increasing the seismic resistance of underground pipelines, mitigating seismic effect, and possible damage during earthquakes are of great importance worldwide. Improving the seismic resistance, reliability, and safety of pipeline transport is an urgent task. Pipelines laid in difficult geotechnical conditions experience such loads as the weight of the pipeline conveying a product, the weight of soil surrounding the pipeline, the response of soil to the pipeline strain, internal working pressure and temperature stresses, the loads related to the changes in physical and mechanical characteristics of the soil. To avoid pipeline failures and accidents, it is necessary to establish the influence of design features, the changes in operating conditions, and parameters on the strength and stability of the pipeline.

To ensure the earthquake resistance of the above-ground and underground sections of the pipeline, it is necessary to study its stress-strain state (SSS), taking into account the naturalclimatic loads and the operating parameters and design features of the pipeline, the influence of various forms of bending.

The grounds of the dynamic theory of earthquake resistance of complex systems of underground pipelines were developed in [1-3]. It was assumed that any structure is elongated, branching one both along the strike and in-depth, with complex rigid and flexible pipe joints in a complex node. In [4-13], the seismodynamics of the underground structures under the harmonic and real seismic impacts are numerically studied.

*Corresponding author: nematilla81@mail.ru 
In [14], the principles of calculation and design of underground structures in seismic regions were considered. The stresses at the underground pipeline bend investigated experimentally. It was determined that when two pipelines are connected at a right angle in a node, its displacements are approximately equal to the displacements of the surrounding soil. As a result, axial forces and bending moments appear in the curved sections of the pipelines during an earthquake. The test results showed that the axial forces were initiated in the pipeline in the direction of forced vibrations, and the bending moments appear at the right angle junction of two pipelines.

When solving the problems of seismodynamics of underground pipelines, the main issue is the interaction modeling in the pipe-soil system. In this paper, we proposed a calculation method to determine the SSS of a polymeric L-shaped pipeline (figure 1), subjected to seismic effects, the front of which forms angle $\alpha$ with the axis $O x$ directed along the pipeline $I$ axis. The results of theoretical studies allow estimating the stress-strain state of L-shaped polymeric pipelines under seismic load, directed arbitrary relative to the principal axes of the structure (figure 1) [15]. Recently, much attention has been paid to the calculation of pipelines of various configurations (L-, T-, $\mathbf{U}$ - and $\mathbf{V}$-shaped ones) [15].

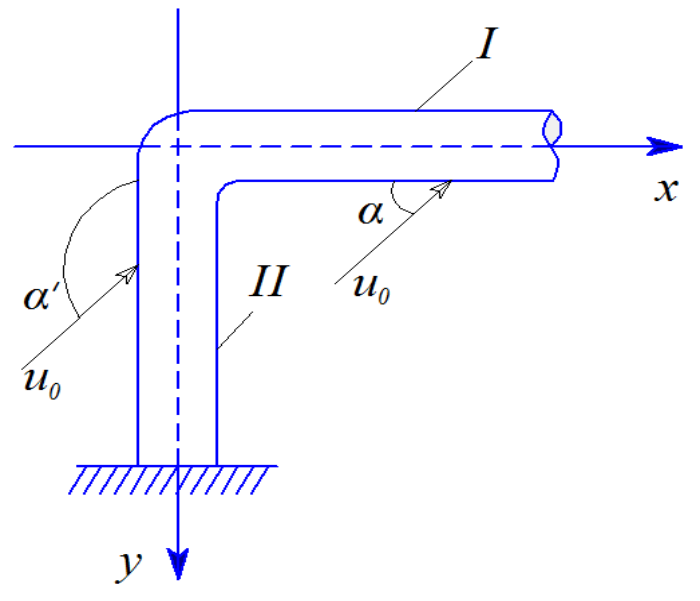

Fig. 1. L-shaped pipeline section in the plane: I is pipeline; II is pipeline

\section{Methods}

To derive a system of differential equations of motion with boundary and initial conditions, the Hamilton - Ostrogradsky variational principle was used [16-18]

$$
\int_{t}(\delta \mathrm{T}-\delta \Pi+\delta \mathrm{A}) d t=0
$$

where $\delta T, \delta \Pi$ are the variations of kinetic and potential energy, $\delta A$ is the variation of work of external forces, $t$ is time.

Consider the forces and moments of two pipelines $I$ and $I I$ connected in a node

$$
N_{x I}=Q_{12 I I}, N_{x I I}=Q_{12 I}, M_{x I}=M_{x I I}
$$

The stresses-strains relationship for underground polymeric pipelines are obtained from [19] 


$$
\begin{gathered}
\sigma_{11}=E\left[\varepsilon_{11}(t)-\int_{0}^{t} \Gamma(t-\tau) \varepsilon_{11}(\tau) d \tau\right],\left(\begin{array}{l}
I \rightarrow I I \\
x \rightarrow y
\end{array}\right) \\
\sigma_{12}=G\left[\varepsilon_{12}(t)-\int_{0}^{t} \Gamma(t-\tau) \varepsilon_{12}(\tau) d \tau\right],\left(\begin{array}{l}
I \rightarrow I I \\
x \rightarrow y
\end{array}\right),
\end{gathered}
$$

where $G=\frac{E}{2(1+\mu)}$, as $I \rightarrow x$ for the first pipeline, as $I I \rightarrow y$ for the second pipeline, the equations are written iteratively (see figure 1).

Considering relationships (3), the system of equations (1) in displacements has the form:

$$
\begin{aligned}
& -\rho F \frac{\partial^{2} u}{\partial t^{2}}+E F\left(\frac{\partial^{2} u}{\partial x^{2}}-\int_{0}^{t} \Gamma(t-\tau) \frac{\partial^{2} u}{\partial x^{2}} d \tau\right)+N_{x}\left(P_{1}\right)+N_{x}\left(q_{1}\right)=0, \quad\left(\begin{array}{l}
I \rightarrow I I \\
x \rightarrow y
\end{array}\right), \\
& -\rho F \frac{\partial^{2} \mathrm{v}}{\partial t^{2}}+G F\left(\left(\frac{\partial^{2} \mathrm{v}}{\partial x^{2}}-\frac{\partial \alpha_{1}}{\partial x}\right)-\int_{0}^{t} \Gamma(t-\tau)\left(\frac{\partial^{2} \mathrm{v}}{\partial x^{2}}-\frac{\partial \alpha_{1}}{\partial x}\right) d \tau\right)+Q\left(P_{2}\right)+Q\left(q_{2}\right)=0,\left(\begin{array}{l}
I \rightarrow I I \\
x \rightarrow y
\end{array}\right), \\
& -\rho I_{z} \frac{\partial^{2} \alpha_{1}}{\partial t^{2}}+\left(E I_{z} \frac{\partial^{2} \alpha_{1}}{\partial x^{2}}+G F \frac{\partial^{2} \mathrm{v}}{\partial x^{2}}-G F \alpha_{1}\right)-\int_{0}^{t} \Gamma(t-\tau)\left(E I_{z} \frac{\partial^{2} \alpha_{1}}{\partial x^{2}}+G F \frac{\partial^{2} \mathrm{v}}{\partial x^{2}}-G F \alpha_{1}\right) d \tau- \\
& -\left(M_{z}\left(P_{1}\right)+M_{z}\left(q_{1}\right)\right)=0, \quad\left(\begin{array}{l}
I \rightarrow I I \\
x \rightarrow y
\end{array}\right) .
\end{aligned}
$$

The boundary conditions are:

$$
\begin{aligned}
& {\left.\left[-E F\left(\frac{\partial u}{\partial x}-\int_{0}^{t} \Gamma(t-\tau) \frac{\partial u}{\partial x} d \tau\right)+N_{x}\left(\varphi_{1}\right)\right] \delta u\right|_{x}=0, \quad\left(\begin{array}{l}
I \rightarrow I I \\
x \rightarrow y
\end{array}\right),} \\
& {\left.\left[-G F\left(\left(\frac{\partial \mathrm{v}}{\partial x}-\alpha_{1}\right)-\int_{0}^{t} \Gamma(t-\tau)\left(\frac{\partial \mathrm{v}}{\partial x}-\alpha_{1}\right)\right)+Q_{12}\left(\varphi_{2}\right)\right] \delta \mathrm{v}\right|_{x}=0,\left(\begin{array}{l}
I \rightarrow I I \\
x \rightarrow y
\end{array}\right),} \\
& {\left.\left[E I_{z}\left(\frac{\partial \alpha_{1}}{\partial x}-\int_{0}^{t} \Gamma(t-\tau) \frac{\partial \alpha_{1}}{\partial x} d \tau\right)+M_{z}\left(\varphi_{1}\right)\right] \delta \alpha_{1}\right|_{x}=0,\left(\begin{array}{l}
I \rightarrow I I \\
x \rightarrow y
\end{array}\right) .}
\end{aligned}
$$

Here, for the pipe $I$, the boundary conditions are formed at the junction of pipelines $I$ and $I I$. The boundary conditions (5) and relationships are used, where the junction node of pipelines $I$ and $I I$ is indicated by $B$.

$$
N_{x}\left(\varphi_{1}\right)=R_{x}^{B}=q_{1}^{I} l^{I}, Q_{12}\left(\varphi_{2}\right)=R_{y}^{B}=q_{2}^{I} l^{I}, M_{z}\left(\varphi_{1}\right)=M_{z}^{B}=\frac{1}{2} q_{2}^{I}\left(l^{I}\right)^{2} .
$$

The initial conditions for pipelines $I$ and $I I$ (figure 1) have the form:

$$
\left.\rho F \frac{\partial u}{\partial t} \delta u\right|_{t}=0,\left.\rho I_{z} \frac{\partial \alpha_{1}}{\partial t} \delta \alpha_{1}\right|_{t}=0,\left.\rho F \frac{\partial v}{\partial t} \delta v\right|_{t}=0
$$


Here $q_{1}^{I}, q_{2}^{I}$ are the seismic forces acting on pipelines $I$ and $I I$ (Fig. 1); they have the form (see monographs [14]):

$$
\begin{aligned}
& N_{x}\left(q_{1}\right)=-\pi D_{H} k_{x}\left(u-u_{0 x}\right), Q_{12}\left(q_{2}\right)=-2 p \pi D_{H} k_{x}\left(\mathrm{v}-u_{0 y}\right), \\
& M_{z}\left(q_{1}\right)=q\left(\alpha_{1}-\frac{\partial u_{0 y}}{\partial x}\right), q=\frac{5}{\bar{\beta} p}\left(\frac{l}{D_{H}}\right)^{3} R_{H}^{3} k_{x}, \quad p=\left(1+\mu_{\text {zрунш }}\right), \bar{\beta}=\left(1-\frac{D_{B}^{4}}{D_{H}^{4}}\right),
\end{aligned}
$$

where $\mu_{\text {soil }}$ is the Poisson's ratio of soil, $k_{x}$ is the coefficient of longitudinal interaction with soil, $l$ is the length that corresponds to experimental study [12], $u_{0 x}$ and $u_{0 y}$ are the laws of ground motion.

If we take into account relationships (8), then the system of equations (4) takes the form

$$
\begin{gathered}
-\rho F \frac{\partial^{2} u}{\partial t^{2}}+E F\left(\frac{\partial^{2} u}{\partial x^{2}}-\int_{0}^{t} \Gamma(t-\tau) \frac{\partial^{2} u}{\partial x^{2}} d \tau\right)-\pi D_{H} k_{x}\left(u-u_{0 x}\right)=0, \quad\left(\begin{array}{l}
I \rightarrow I I \\
x \rightarrow y
\end{array}\right), \\
-\rho F \frac{\partial^{2} v}{\partial t^{2}}+G F\left(\left(\frac{\partial^{2} v}{\partial x^{2}}-\frac{\partial \alpha_{1}}{\partial x}\right)-\int_{0}^{t} \Gamma(t-\tau)\left(\frac{\partial^{2} v}{\partial x^{2}}-\frac{\partial \alpha_{1}}{\partial x}\right) d \tau\right)+\left(-2 p \pi D_{H} k_{x}\left(v-u_{0 y}\right)\right)=0,\left(\begin{array}{l}
I \rightarrow I I \\
x \rightarrow y
\end{array}\right), \\
-\rho I_{z} \frac{\partial^{2} \alpha_{1}}{\partial t^{2}}+\left(E I_{z} \frac{\partial^{2} \alpha_{1}}{\partial x^{2}}+G F \frac{\partial v}{\partial x}-G F \alpha_{1}\right)-\int_{0}^{t} \Gamma(t-\tau)\left(E I_{z} \frac{\partial^{2} \alpha_{1}}{\partial x^{2}}+G F \frac{\partial v}{\partial x}-G F \alpha_{1}\right) d \tau- \\
-\left(q\left(\alpha_{1}-\frac{\partial u_{0 y}}{\partial x}\right)\right)=0, \quad\left(\begin{array}{l}
I \rightarrow I I \\
x \rightarrow y
\end{array}\right) .
\end{gathered}
$$

Proceed to dimensionless displacements and coordinates

$$
u=\bar{u} \cdot D_{H}, u_{0}=\bar{u}_{0} \cdot D_{H}, v=\bar{v} \cdot D_{H}, x=\bar{x} \cdot l, t=\bar{t} \cdot t_{0} .
$$

In calculations, the weakly singular three-parameter Rzhanitsyn-Koltunov kernel [19] was used in expressions (5) and (9),

$$
\Gamma(t)=\bar{A}_{b} e^{-\bar{\beta} t} t^{\bar{\alpha}-1}, 0<\alpha<1,[\bar{\beta}]=c^{-1},\left[\bar{A}_{b}\right]=c^{-\bar{\alpha}} .
$$

The three-parameter kernel (11) has a weak Abel-type singularity. The kernels of this type have a weak singularity. To eliminate it, the transforms in the integrand were conducted, according to [20].

After introducing the vectors of external force displacements, we performed some transforms, then from the system of equations (9), we obtained a differential equation of motion, boundary, and initial conditions in a general vector form

$$
\begin{gathered}
M \frac{\partial^{2} Y}{\partial t^{2}}+A \frac{\partial^{2} Y}{\partial x^{2}}-\frac{A_{b}}{\bar{\alpha}} \sum_{k=1}^{n} B_{k}^{b} e^{-\beta t_{k}} A \frac{\partial^{2} Y\left(t_{n}-t_{k}\right)}{\partial x^{2}}+B \frac{\partial Y}{\partial x}- \\
-\frac{A_{b}}{\bar{\alpha}} \sum_{k=1}^{n} B_{k}^{b} e^{-\beta t_{k}} B \frac{\partial Y\left(t_{n}-t_{k}\right)}{\partial x}+C Y-\frac{A_{b}}{\bar{\alpha}} \sum_{k=1}^{n} B_{k}^{b} e^{-\beta t_{k}} D Y\left(t_{n}-t_{k}\right)=Q,\left(\begin{array}{l}
I \rightarrow I I \\
\bar{x} \rightarrow \bar{y}
\end{array}\right)
\end{gathered}
$$

where $Q=\left[\frac{l^{2} b^{2}}{a_{T}^{2}} \bar{u}_{0 x} \frac{q}{E F} \frac{\partial \bar{u}_{0 z}}{\partial \bar{x}} \frac{2 p l^{2} b^{2}}{a_{T}^{2}} \bar{u}_{0 z}\right]^{T}, Y=\left\{u, \mathrm{v}, \alpha_{1}\right\}^{T}, M, A, B, C$ are the third-order matrices (figure 1 , as $I \rightarrow x$ for the first pipeline, as $I I \rightarrow y$ for the second pipeline, the equations are written iteratively). 
Boundary conditions in a vector dimensionless form for the pipe $I$ are:

$$
\begin{array}{r}
{\left.\left[\bar{A} \frac{\partial Y}{\partial x}-\frac{A_{b}}{\bar{\alpha}} \sum_{k=1}^{n} B_{k}^{b} e^{-\beta t_{k}} \bar{A} \frac{\partial Y\left(t_{n}-t_{k}\right)}{\partial x}+\bar{B} Y-\frac{A_{b}}{\bar{\alpha}} \sum_{k=1}^{n} B_{k}^{b} e^{-\beta t_{k}} \bar{B} Y\left(t_{n}-t_{k}\right)+\bar{C}\right]\right|_{\bar{x}=0}=0} \\
{\left.\left[\bar{A}_{1} \frac{\partial Y}{\partial x}-\frac{A_{b}}{\bar{\alpha}} \sum_{k=1}^{n} B_{k}^{b} e^{-\beta t_{k}} \bar{A}_{1} \frac{\partial Y\left(t_{n}-t_{k}\right)}{\partial x}+\bar{B}_{1} Y-\frac{A_{b}}{\bar{\alpha}} \sum_{k=1}^{n} B_{k}^{b} e^{-\beta t_{k}} \bar{B}_{1} Y\left(t_{n}-t_{k}\right)\right]\right|_{\bar{x}=l}=0}
\end{array}
$$

for pipe $I I$ are:

$$
\begin{gathered}
{\left.\left[\overline{\bar{A}} \frac{\partial Y}{\partial x}-\frac{A_{b}}{\bar{\alpha}} \sum_{k=1}^{n} B_{k}^{b} e^{-\beta t_{k}} \overline{\bar{A}} \frac{\partial Y\left(t_{n}-t_{k}\right)}{\partial x}+\overline{\bar{B}} Y-\frac{A_{b}}{\bar{\alpha}} \sum_{k=1}^{n} B_{k}^{b} e^{-\beta t_{k}} \overline{\bar{B}} Y\left(t_{n}-t_{k}\right)+\overline{\bar{C}}\right]\right|_{\bar{y}=0}=0} \\
\left.U\right|_{\bar{y}=1}=0
\end{gathered}
$$

where $\bar{A}, \bar{A}_{1}, \overline{\bar{A}}, \bar{B}, \bar{B}, \overline{\bar{B}}, \bar{C}, \overline{\bar{C}}$ are the third-order matrices. Initial conditions are:

$$
\left.\frac{\partial Y}{\partial \bar{t}} \delta Y\right|_{t}=0 .
$$

To solve the boundary value problem (12), (13), and (14), we use the method of finite differences of the second order of accuracy.

The problem is solved based on the algorithm of computer implementation. Mechanical and geometrical parameters are selected as follows: $E=5 \cdot 10^{2} \mathrm{MPa} ; \rho=940 \mathrm{~kg} / \mathrm{m}^{3}$; $D_{B}=0.392 \mathrm{~m} ; \quad D_{H}=0.4 \mathrm{~m} ; \quad F=\pi\left(D_{H}^{2}-D_{B}^{2}\right) / 4 \mathrm{~m}^{2} ; \quad I_{z}=\pi\left(D_{H}^{2}-D_{B}^{2}\right) / 64 \mathrm{~m}^{4} ; \quad l^{I}=l^{I I}=20 \mathrm{~m} ;$ $k_{x}=1.2 \cdot 10^{4} \mathrm{kN} / \mathrm{m}^{3} ; u_{0 x}(x, t)=A \sin \omega t^{*} \cos \alpha ; a=0.004 \mathrm{~m} ; \varepsilon=0.3 \mathrm{~s}^{-1} ; \omega=2 \pi / T ; T=0.3 \mathrm{~s} ; \mu_{\text {soil }}=0.2$; $\mu_{\text {pipe }}=0.3 ; \mathrm{A}_{\mathrm{b}}=0.1 ; \bar{\alpha}=0.25 ; \beta=0.05 ; C_{p}=1500 \mathrm{~m} / \mathrm{s} ; \alpha^{\prime}=\alpha+90$.

\section{Results and Discussion}

Numerical results are obtained for the displacements and force factors considering boundary conditions. The results are presented in graphs. When calculating the pipeline for earthquake resistance, the options were used for setting the ground motion during earthquakes in the form of a sinusoid (harmonic law).

The changes in longitudinal $u$ (figure 2,a) and transverse $v$ (figure 2,b) displacements of underground pipelines at time $t$ at the point of their intersection $(x=0, y=0)$ at the incidence angle $\alpha=30^{\circ}$ of seismic load are given below. 


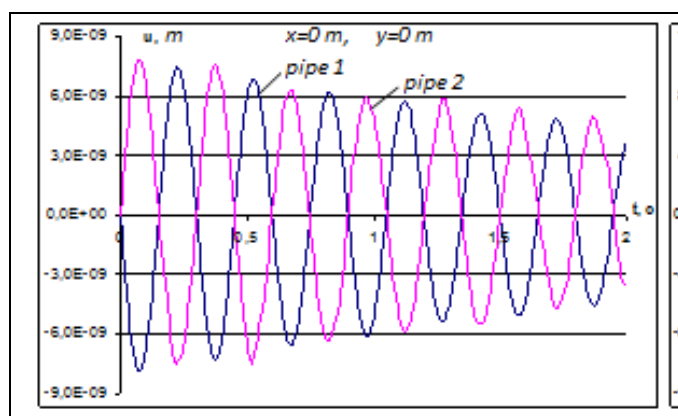

a)

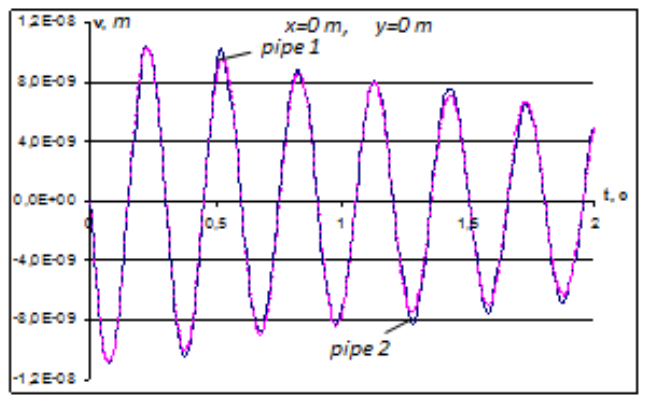

b)

Fig. 2. Changes in longitudinal and transverse displacements of pipelines $I$ and $I I$ in time at their intersection point

As seen from figure 2, at the junction point of pipelines $I$ and $I I$, the values of longitudinal displacements change similarly to each other, but the vibration amplitudes have a negative sign. And for the transverse displacements of pipelines $I$ and $I I$ at the point of their junction, the vibration amplitudes in phases coincide with each other (figure 2, b).

Figure 3, a shows the change in longitudinal displacement of a polymeric pipeline at the point $x=0 \mathrm{~m}$ in time at different angles of incidence of seismic load. The maximum displacement of the pipeline occurs at $\alpha=0$, with a decrease in the angle of incidence, the values of the maximum displacement decrease. Over time, the amplitude of vibrations also decreases.

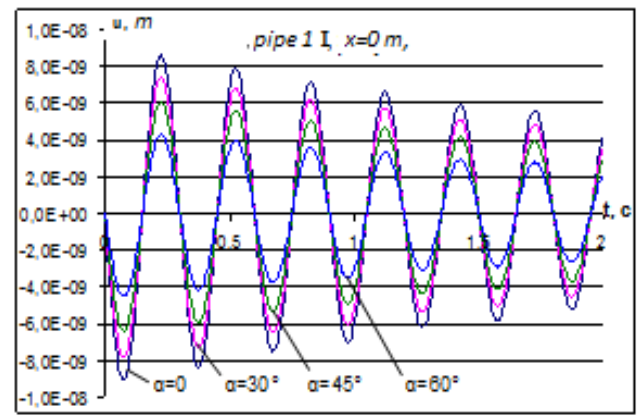

a)

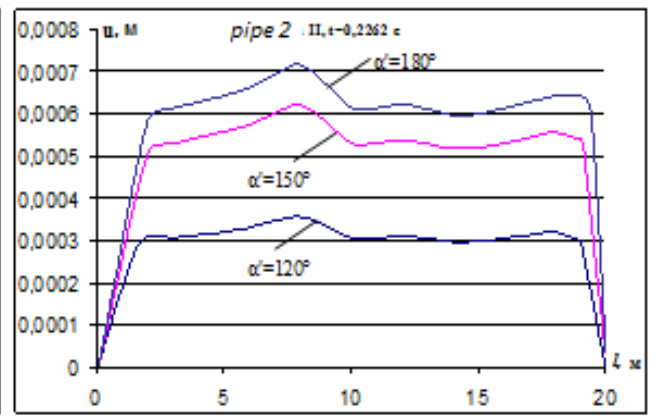

b)

Fig. 3. Change in longitudinal displacement of the pipeline in time at the point of pipelines intersection and along the axis at fixed times at different angles of incidence of seismic load

In pipeline $I I$, the maximum values of longitudinal displacement are reached in the middle of the pipeline (figure 3, b). The values of the angles of incidence of seismic load vary within $120 \leq \alpha^{\prime} \leq 180$ for pipeline $I I$. 


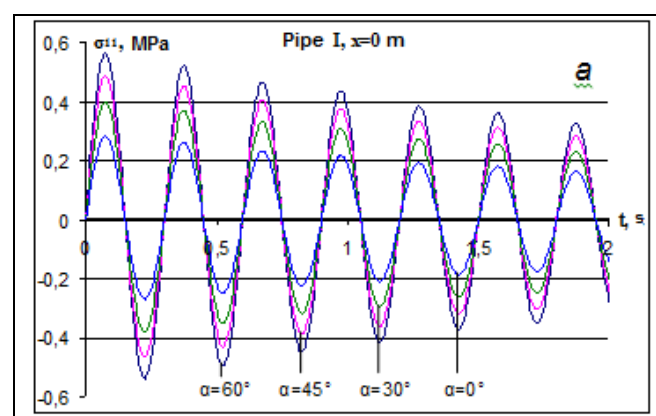

a)

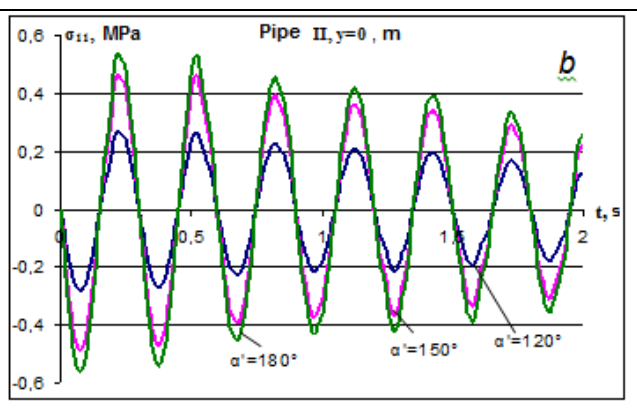

b)

Fig. 4. Changes in longitudinal stresses over time at the point of intersection of pipelines under seismic load at different angles of incidence

Figure 4, a and 4, b shows the changes in longitudinal stresses over time at the intersection of pipelines $I$ and $I I(x=0, y=0)$ under seismic load at different angles of incidence. With a decrease in the angle of incidence of seismic load in the pipe $I$, the longitudinal stress values increase, the vibration phases coincide within $0 \leq \alpha \leq 90$ (figure 4). In pipeline $I I$, with an increase in the angle of incidence of seismic load, the longitudinal stress values also increase.

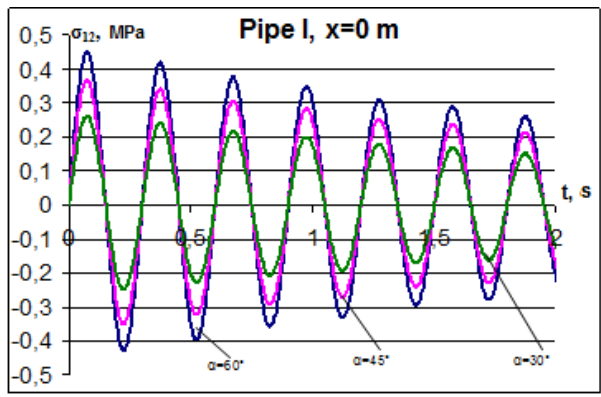

a)

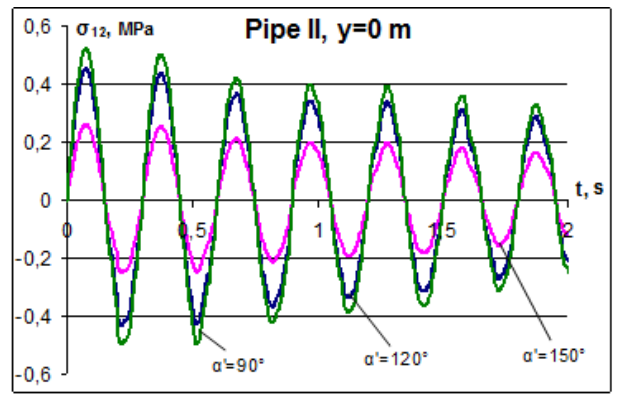

b)

Fig. 5. Changes in shear stresses of pipelines over time at the point of intersection of pipelines under seismic load at different angles of incidence

Figure 5 shows the changes in shear stress values of pipelines I and II in time at the point of their intersection under seismic load at different angles of incidence. From the figures, it can be seen that the maximum value of shear stress in both pipes is reached at $\alpha^{\prime}=90$. With a change in the angle of incidence of seismic load, the vibration phases of shear stress in both pipes do not change.

\section{Conclusions}

Thus, the simultaneous equations of longitudinal and transverse vibrations of an underground polymeric pipeline of the L-shaped configuration were derived for an arbitrary direction of seismic load. A computational scheme of the problem was constructed using central finite-difference relations. The analysis of the results obtained on the simultaneous longitudinal and transverse vibrations of underground L-shaped polymeric pipelines under seismic loading was carried out. The SSS of the L-shaped polymeric pipeline subjected to seismic effect was determined, and the axial forces and bending moments arising in curved pipelines during an earthquake were determined. An analysis of the above problems shows 
that the viscoelastic properties of the polymeric pipeline contribute to the mitigation of external seismic effect, which consequently leads to less damage and thereby increases the strength and stability of these structures.

\section{References}

1. Rashidov T.R., Mubarakov Y. Seismodynamics of underground structures, Soil Mech. Found. Eng. 29 pp. 213-216 (1992)

2. Rashidov T.R. Dynamic theory of earthquake resistance of complex systems of underground structures, Tashkent, "Fan", (1973)

3. Rashidov T, Ishankhodzhaev A .A., and Abduzhabarov A . Earthquake effects on the shallow lying tunnels and earth road bed (1973)

4. Bekmirzaev D.A., Mirzaev I. Earthquake Resistance Assessment of Buried Pipelines of Complex Configuration Based on Records of Real Earthquakes, Soil Mech. Found. Eng. pp. 491-496, (2021)

5. Bekmirzaev D.A., Mirzaev I., Mansurova N Sh., Kosimov E.A., and Juraev D. Numerical methods in the study of seismic dynamics of underground pipelines, IOP Conf. Ser. Mater. Sci. Eng. 869052035 (2020)

6. Bekmirzaev D.A., Kishanov R.U., and Mansurova N.Sh. Mathematical Simulation and Solution of the Problem of Seismo-Dynamics of Underground Pipelines, Int. J. Emerg. Tr. Eng. Res. 8 pp. 5028-5033, (2020)

7. Bekmirzaev D.A., Mansurova N.Sh., Nishonov N.A., Kosimov E.A., and Numonov A. Underground pipelines dynamics problem solution under longitudinal seismic loading, IOP Conf. Ser. Mater. Sci. Eng., 883, 012045, (2020)

8. Nishonov N.A., Bekmirzaev D.A., An E.V., Urazmukhamedova Z and Turajonov K. Behaviour and Calculation of Polymer Pipelines Under Real Earthquake Records, IOP Conf. Ser. Mater. Sci. Eng. 869, 052076 (2020)

9. Kosimov E.A., Mirzaev I and Bekmirzaev D.A. Comparison of the impacts of harmonic and seismic waves on an underground pipeline during the Gazli earthquake, IOP Conf. Ser. Mater. Sci. Eng. 1030, 012082, (2021)

10. Miralimov M, Adilov F, Abirov R, Juraev D and Khazratkulov I. To numerical approach for calculation of underground structures, IOP Conf. Ser. Mater. Sci. Eng. 883, 012204 (2020)

11. Amanov A.T, Bahadirov G.A, Tsoy G.N and Nabiev A.M. A New Method to Wring Water-Saturated Fibrous Materials, Int. J. Mech. Eng. Robot. Res. 10 pp. 151-156, (2021)

12. Rashidov T.R., and Nishonov N.A. Seismic Behavior of Underground Polymer Piping with Variable Interaction Coefficients, Soil Mech. Found. Eng. (2016)

13. Rashidov T.R., Mardonov B.M. and An E.V. Transverse Vibrations of Buried Pipelines Under Axial Loading Within Geometrically Nonlinear Theory, Int. Appl. Mech. 55 pp. 229-238, (2019)

14. Rashidov T.R. and Khojmetov G.X. Earthquake resistance of underground pipelines, Tashkent, "Fan" (1985)

15. Bekmirzaev D.A. and Mirzaev I. Dynamic processes in underground pipelines of complex orthogonal configuration at different incidence angles of seismic effect, Int. J. Sci. Technol. Res. 9 2449-2453, (2020)

16. Kabulov V.K. Algorithmization in the theory of elasticity and strain plasticity, A monograph. Tashkent, Fan. p. 386, (1966)

17. Rashidov T.R., Yuldashev T and Bekmirzaev D.A. Seismodynamics of Underground Pipelines with Arbitrary Direction of Seismic Loading, Soil Mech. Found. Eng. 55 pp. 
243-248 (2018)

18. Rashidov T.R. and Bekmirzaev D.A. Seismodynamics of Pipelines Interacting with the Soil Soil Mech. Found. Eng., 52, pp. 149-54, (2015)

19. Ilyushin A.A. Plasticity. Elastic-plastic strains. Part 1. M.: Logos,. p. 388, (2004)

20. Badalov F.B. Methods for solving integral and integro-differential equations of the hereditary theory of viscoelasticity, A monograph, p. 272, Tashkent, (1987) 